\title{
Die Pflanzenwelt der Höhlen bei Lillafüred
}

\author{
Von Klara Verseghy ${ }^{1}$ ) \\ Mit Tafel 106 (1) und 2 Abbildungen im Text
}

\section{Einleitung}

Im östlichen Teil des Bükk-Gebirges, in einem tiefen, zerklüfteten Tal liegt einer der schönsten Höhenkurorte Ungarns: Lillafüred, verborgen. Dieses an Karstphänomenen reiche Gebiet enthält zahlreiche Höhlen.

Im Frühling des Jahres 1964 habe ich zwei von diesen Lillafüreder Höhlen besucht, um ihre Pflanzenwelt zu erforschen und kennenzulernen: die Forrás-Höhle, die nahe dem Palota-Hotel liegt, und die Istৎán-Höhle, die etwa $700 \mathrm{~m}$ von der ersten entfernt liegt. Wir besitzen zur Zeit nur spärliche Angaben über die lebenden Organismen und besonders über die Pflanzenwelt vieler bekannter Höhlen Ungarns. Unsere Forscher haben die Höhlen größtenteils nur bis zur Tageslichtgrenze erforscht (Boros, 1935; Kol, 1957).

Über die Vegetation der Höhlen wird nur im allgemeinen geschrieben, zum Beispiel Gebhardt (1934): ,In der Höhle von Abaliget (Mecsek-Gebirge) kommen grünende Pflanzen (Lebermoos, Laubmoos, Farn) bloß im Höhlenhals vor." - Jakucs (1952): ,Es leben dort Eisenbakterien und Schwefelbakterien; auf dem Holzmaterial finden wir überall Pilzlager."

Im vorigen Jahrzehnt haben Claus (1955, 1959, $1962 \mathrm{a}, \mathrm{b})$, Suba (1957) und Palik $(1960 \mathrm{a}, \mathrm{b})$ in einigen ungarischen Höhlen algologische Forschungen vorgenommen (in den Baradla-, Abaliget-, Békeund Pálvölgy-Höhlen). Im Laufe dieser Forschungen sind in diesen Höhlen zahlreiche Algenarten gefunden worden (z.B. in der BékeHöhle 90 Arten).

Der Eingang zur Forrás-Höhle bei Lillafüred befindet sich neben dem letzten Wasserfall des Garadna-Baches, in einer Höhe von $264 \mathrm{~m}$ ü.d.M. Die Entdeckung dieser Höhle fällt vermutlich in die Jahre um 1770. Sie ist für Besucher im Jahre 1927 eröffnet; infolgedessen war

1) Botanische Abteilung des Naturhistorischen Museums, Budapest, Ungarn. 
auch die Einführung des elektrischen Lichtes notwendig geworden. Wir können die Ansiedlung der sekundären Vegetation (z. B. Moose) von diesem Zeitpunkt an datieren.

Die Forrás-Höhle unterscheidet sich von den üblichen Karsthöhlen. Ihre Aushöhlungen sind nicht durch die korrodierende und erodierende Wirkung des Wassers im Karstgestein entstanden, sondern bildeten sich gleichzeitig mit dem sich ablagernden Kalktuff. Die etwa $40 \mathrm{~m}$ dicke Kalktuffbank hat sich Ende des Pleistozän von dem aus dem höher gelegenen Szinva-Tal herunterstürzenden, aus der Szinva-Quelle und aus der vermutlich damals noch aktiven István-Höhle hervorquellenden kalkhaltigen Karstwasser - infolge Verminderung seines Kohlendioxydgehaltes - abgelagert.

Der Eingang zur István-Höhle öffnet sich neben der Landstraße zwischen Lillafüred und Eger in einer Höhe von $318 \mathrm{~m}$ ü.d.M. Diese Höhle hat sich im Kalkstein vom Mitteltrias gebildet und verdankt ihre Entstehung einem unterirdischen Wasserlauf. Dieser unterirdische Bach hat vermutlich das Niederschlagswasser der Hochebene des Bükk-Gebirges abgeleitet und ist dann in Verbindung mit der Senkung der Talsohle auf ein niedrigeres Niveau gelangt. In der Ausbildung des Höhlensystems spielen mehrere Faktoren: tektonische, Korrosions- und Erosionswirkungen sowie der hydrostatische Druck des Karstwassers usw., eine Rolle. Die István-Höhle ist eine charakteristische Tropfsteinhöhle mit einem Etagesystem. Der tiefste Punkt liegt in $294 \mathrm{~m}$ ü.d.M., der höchste in einer Höhe von $336 \mathrm{~m}$ ü.d.M. Seit 1931 ist sie für das Publikum geöffnet; damals hat man auch die Elektrizität eingeführt (Vegetation!). (Siehe auch die beigelegten Kartenskizzen.)

Das Ziel meiner Forschungen war, festzustellen: 1. Was für eine Vegetation lebt in diesen Höhlen? 2. Was für ökologische Verhältnisse ermöglichen für sie das Leben hierselbst? 3. Können wohl diese Pflanzen in irgendeiner Weise die Luft dieser Höhlen beeinflussen?

\section{Resultate}

Meine Forschungen habe ich in beiden Höhlen nicht beim Eingang derselben oder in dessen Nähe, sondern davon entfernt begonnen, damit das gesammelte Material ungetrübt von jeder äußeren ökologischen Einwirkung, hauptsächlich des Tageslichtes, sei und nur die wahrhaft im Inneren der Höhle lebende und die daselbst herrschenden eigenartigen Verhältnisse vertragende Vegetation widerspiegele.

1. In der Forrás-Höhle begann ich meine Sammlung im ,,Tripeltor". Unmittelbar neben der Lampe fand ich ein blühendes Mooslager, 


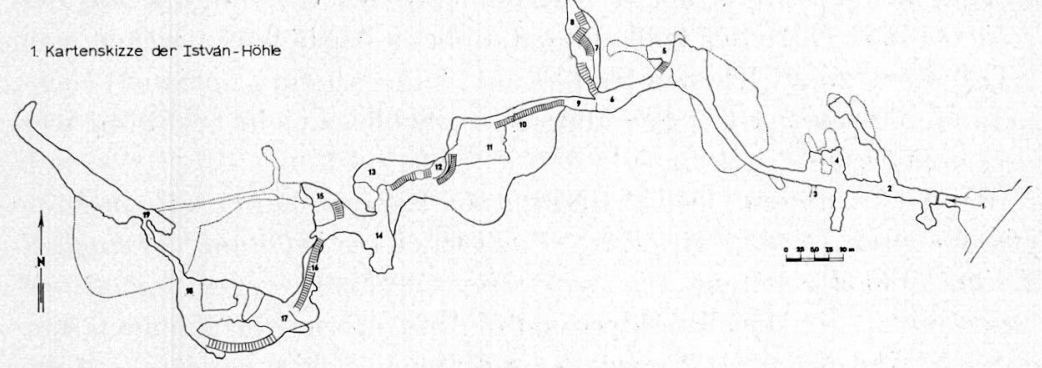

Abb. 1

1. Kartenskizze der István-Höhle: 1. Eingang, 2. Büro, 3. Schacht I, 4. Schacht II, 5. Großer Saal, 6. Entdeckungszweig ,,Tordai Kluft“, 7. Treppenaufgang, 8. Gloriette, 9. Treppenabsatz. - Große Kuppelhalle: 10. Märchenland, 11. Riesiger Wasserfall, 12. Bastei, 13. Basteidurchweg, 14. Säulensaal, 15. Theatersaal, 16. Bergwerkstollen, 17. Schmaler Saal, 18. Hintere Halle, 19. Riesentorte.

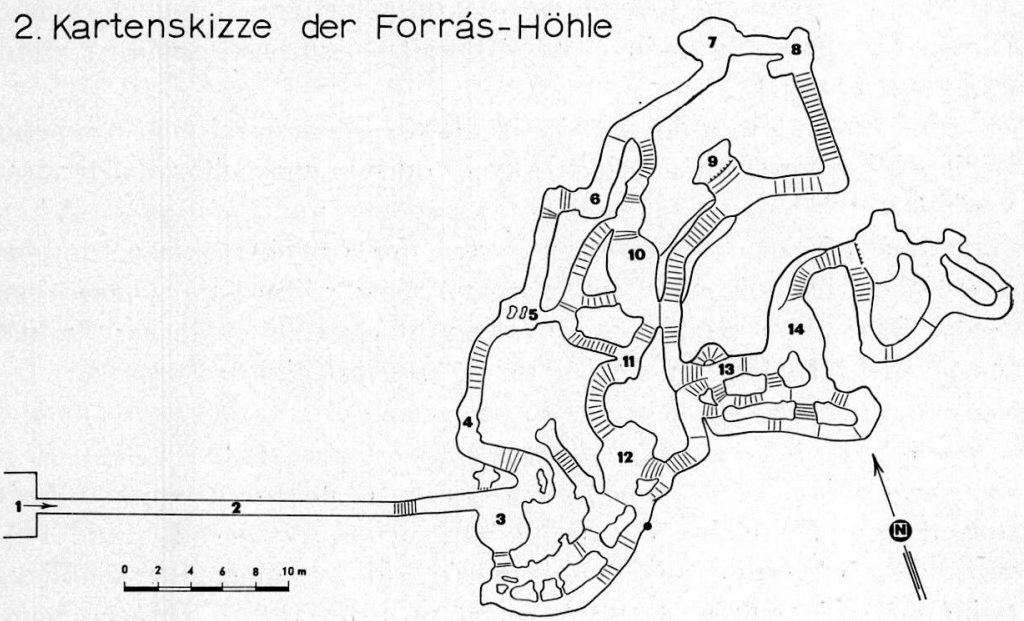

Abb. 2

2. Kartenskizze der Forrás-Höhle: 1. Eingang, 2. Künstlicher Stollen, 3. Halle, 4. Ausgebuchteter Gang, 5. Tripeltor, 6. Untere Quelle, 7. Nordlichtsaal, 8. Tropfsteintor, 9. Herzsaal, 10. Trauerweidensaal, 11. Kleiner Saal, 12. Tropfsteinsaal, 13. Hölle, 14. Verzaubertes Kastell. 
bestehend aus Eucladium verticillatum (With.) Br. Eur. var.angustifolium Lindb. ${ }^{2}$ ). In der vollkommen dunklen Aushöhlung, welche vom ,Tripeltor" zum ,Kleinen Saal" führt, habe ich ein Coprinus ${ }^{2}$ )-Lager gefunden, dessen Art wegen Mangels an Schleier nicht bestimmt werden konnte.

Bei der ,Unteren Quelle" sind um die Lampen herum: Rhynchostegium murale (Neck.) Br. Eur., Eucladium verticillatum var.angustifolium, und der seltene Fissidens minutulus Sull.; gegen den ,Nordlichtsaal" zu in der Korridorwendung auf einem verfaulenden Türrahmen sind bräunlichgrauen Belag bildende Schimmelpilze, auf den Treppen dahinter ist ein Coprinus-Lager. Um die Lampen herum: Eucladium verticillatum var. angustifolium und Pohlia sp. Im ,Nordlichtsaal" bedecken in 200, $250 \mathrm{~cm}$ Höhe um die Lampen herum hellgrüne Moospolster an einer Stelle $16 \mathrm{dm}^{2}$ Fläche, bei der anderen Lampe $1 \mathrm{~m}^{2}$ und bei der dritten $1,5 \mathrm{~m}^{2}$ Fläche. Die hier gefundenen Moosarten sind: Fissidens minutulus Sull., Brachythecium velutinum (L.) Br. Eur., Barbula fallax Hedw., Amblystegium riparium (L.) Br. Eur., Rhynchostegium murale (Neck.) Br. Eur. An dieser Stelle ist eine große Wandfläche auch mit einer einzelligen Cyanophyta bedeckt. In der vom ,,Trauerweidensaal" nördlich gelegenen Strecke ist Eucladium verticillatum (With.) Br. Eur. wiederzufinden. Im ,,Tropfsteinsaal“ ist auf den Wänden ein bräunlicher schimmelartiger Belag zu sehen. Diesen - und im allgemeinen die Mikropilze - zu bestimmen ist nicht möglich gewesen.

2. Die Pflanzenwelt der István-Höhle wird in erster Reihe von einer reichen Moosflora repräsentiert. Algen sind sehr spärlich und artenarm vertreten. Ich habe nur einzellige Cyanophyta- und Chlorophyta-Arten gefunden. Die in den einzelnen Teilen der István-Höhle gefundene Vegetation ist folgende: im ,Großen Saal": Barbula unguiculata Hedw. Moos; im Saal vom ,,Riesigen Wasserfall": Moose: Tortula muralis (L.) Hedw. var.aestiva Brid., Amblystegium serpens (L.) Br. Eur., Eucladium verticillatum, Rhynchostegium murale, Pellia fabbroniana Raddi, Bryoerythrophyllum recurvirostrum (Hedw.) Chen., Bryum caespiticium L. Diese bedecken um die Reflektoren von 500 Watt herum 1-2 $\mathrm{m}^{2}$ Fläche. Die Tropfsteine vom ,Wasserfall“" sind teilweise von Fledermaus-Guano bedeckt, auf welchem sich weißliche Schimmelpilze bilden. Diese können im Frühjahr in regnerischem, feuchtem Wetter gut beobachtet werden, verschwinden jedoch im Sommer, besonders wenn große Trockenheit herrscht. In diesem Saal bedeckt dieser Schimmelpilz - gemeinsam mit einer Polyporacea-auch

$\left.{ }^{2}\right)$ Die Moose wurden von L.Vajda und die Pilze von G. Bohus bestimmt. 
die Holzgeländer. Beim „,Basteidurchweg“", bei der Tropfsteinsäule beleuchtenden Lampe sind 2 Moosarten häufig: Amblystegium serpens und Barbula unguiculata. Interessant, daß hier beim Deckenlicht von 100 Watt keine Pflanze zu finden ist und daß auf dem Plafond stetig Wassersickern und Tröpfeln beobachtet werden kann. Im Saal „Märchenland" sind 5 Moosarten: Barbula unguiculata, Fissidens taxifolius (L.) Hedw., Amblystegium serpens, Eucladium verticillatum und Enrynchium schswartzii (Turn.) Hobbirk, welche eine Fläche von 1,5$2 \mathrm{~m}^{2}$ bedecken. Schließlich ist die Moosflora im ,Theatersaal" am reichsten, mit 7 Arten: Eucladium verticillatum, Bryoerythrophyllum recurvirostrum (Hedw.) Chen., Gymnostomum rupestre Schleich. f. ramosissima Br. Eur., Funaria hygrometrica (L.) Sibth., Tortula muralis (L.) Hedw. und Pohlia sp. Vom ,Theatersaal" einwärts sind um die Lampen herum keine Moose mehr, was dadurch verursacht wird, daß man die Besucher nur bis zum ,,Theatersaal" führt, und weiter im Inneren brennt das elektrische Licht nur sehr selten. Daher habe ich in der ,Hinteren Halle“ nur mehr ein Coprinus und auf dem Holzgeländer nur weiße, sich lange hinschlängelnde Pilzfäden und Schimmel gefunden.

\section{Besprechung}

Das bisher Gesagte zusammenfassend, können wir feststellen, daß die Vegetation der Forrás- und István-Höhlen bei Lillafüred von Algen, Mikro- und Makropilzen und Moose vertreten ist. Die Algen sind in beiden Höhlen einzellige Cyanophyta und Chlorophyta mit geringer Artenzahl (etwa 4-5 Arten). Von den Makropilzen können Tintenpilz- und Feuerschwamm-Arten gefunden werden. Die Aufarbeitung der Mikropilze war nicht möglich. Die Moosflora ist sehr reichlich und kann um das künstliche Licht herum als sekundäre Vegetation betrachtet werden. In der Forrás-Höhle habe ich 7, in der István-Höhle 15 Moosarten gefunden. Zwischen den Moosarten der beiden Höhlen sind nur wenige gemeinsam: Rhynchostegium murale, Eucladium verticillatum und Pohlia sp. Eine seltene und interessante Art kommt in mehreren Teilen der Forrás-Höhle vor: Fissidens minutulus. Woher und auf welche Weise ist wohl diese Moosart in die Höhle geraten, welche in Ungarn kaum unter den im Freien wachsenden Arten zu finden ist? Die Lösung dieser Frage ist selbstverständlich eine Aufgabe für die Bryologen.

Über den Ursprung der Energiequelle, welche zur Abwicklung der Lebensprozesse der ewig in vollkommener Dunkelheit lebenden Höhlenalgen benötigt wird, sind verschiedene Hypothesen aufgestellt 
worden. So können zum Beispiel die Algen die Energie der in den Höhlen vorhandenen verschiedenen Ausstrahlungen verwenden. Jedoch laut den Untersuchungen von Claus (1955) ist die GammaStrahlung in den Baradla- und Béke-Höhlen sehr gering, Beta-Strahlung ist überhaupt nicht wahrnehmbar. Laut Fehér (1954) ist aber eine vermutlich sogenannte Mikrostrahlung möglich.

Die Algenvegetation der Höhlen bei Lillafüred ist kärglich. Es ergibt sich die Frage, warum wohl in diesen Höhlen - wo auch künstliche Lichtquellen vorhanden sind - nur einige wenige Arten dieser als primär zu betrachtenden Pflanzenorganismen aufzufinden sind, wo doch in anderen Höhlen Ungarns eine reiche Algenflora lebt.

Demgegenüber wird die Pflanzenwelt von einer ansehnlichen Zahl von Moosarten vertreten. Für diese dienen die Lampen als Energiequelle. Doch brennen die Lampen nur periodisch, wenn Besucher die Höhlen aufsuchen. Dies bedeutet vom Frühjahr bis zum Herbst täglich etwa 2-5 Stunden Beleuchtung, im allgemeinen mit 100-WattGlühbirnen, in den Scheinwerfern mit 500-Watt-Glühbirnen. Im Winter vermindert sich die Besucherzahl stark, und die Brennzeit der elektrischen Birnen ist sehr kurz.

Nun taucht die Frage auf: Warum genügt auch dieses geringe Licht zur Abwicklung der Lebensprozesse der Moosarten? Besitzen sie eventuell auch andere Energiequellen, und wenn ja, welcherart? Verwenden sie vielleicht die obenerwähnte etwaige Mikrostrahlung? Die bei den Algen angenommenen Energiequellen: Symbiose, heterotrophe Ernährung oder Chemosynthese, können bei den Moosen nicht in Betracht kommen. Vielleicht können sie die kurz andauernde, aber starke Lichtenergie aufspeichern? Alle diese Probleme können erst im Laufe weiterer Forschungen beantwortet werden.

Ein anderes Problem: Wieviel Zeit brauchte diese Moosflora, um sich anzusiedeln und zu verbreiten? Lebt in der István-Höhle eine Vegetation von mehreren Jahrzehnten oder nur von einem Jahrzehnt? Die elektrische Beleuchtungseinrichtung der István-Höhle wurde nämlich während des zweiten Weltkrieges ganz zerstört und erst um 1955 herum wiederhergestellt.

Ich habe festgestellt, daß in diesen Höhlen in die Cryptogam-Pflanzengruppe gehörende Flechten nicht vorhanden sind, nicht einmal Protothallus oder Lepraria-Arten, obzwar die für die Flechten so wichtige Feuchtigkeit hier ständig und genügend ist.

Szabó (1963) hat in beiden Höhlen Mikroklimauntersuchungen durchgeführt und festgestellt, daß die relative Luftfeuchtigkeit in der István-Höhle durchschnittlich $86-96 \%$ beträgt, in niederschlags- 
reichem Wetter um 100\% herum. Seine Messungen in der ForrásHöhle wiesen 97-100\% auf.

Es ist anzunehmen, daß für die Flechten der andere Faktor: das Licht der Lampen bzw. die Beleuchtungszeit, für ihre Lebensprozesse ungenügend ist, deshalb kommen sie hier nicht vor.

Die dritte Frage meiner Forschungen: Können die Pflanzen in irgendeiner Weise die Luft der Höhlen beeinflussen? - muß ich mit einem entschiedenen ,Nein“ beantworten. Die Menge der Pflanzen, die Ausdehnung der von ihnen bedeckten Flächen ist im Verhältnis zur Größe der Höhlen zu gering, folglich kann die Vegetation zur Verbesserung der Höhlenluft wesentlich nichts beitragen.

\section{ZUSAMMENFASSUNG}

Die Vegetation der Forrás- und István-Höhlen bei Lillafüred in Ungarn besteht aus Algen, Mikro- und Makropilzen und Moosen. Die Algen sind in beiden Höhlen einzellige Cyanophyta und Chlorophyta mit geringer Artenzahl. Von den Makropilzen können Tintenpilz- und Feuerschwamm-Arten gefunden werden. Die Aufarbeitung der Mikropilze war nicht möglich. Die Moosflora ist reichlich und kann um das künstliche Licht herum als sekundäre Vegetation betrachtet werden. In der Forrás-Höhle wurden 7, in der István-Höhle 15 Moosarten gefunden. Von den Moosarten der beiden Höhlen sind nur wenige gemeinsam: Rhynchostegium murale, Eucladium verticillatum und Pohlia sp. Eine seltene und interessante Art kommt in mehreren Teilen der Forrás-Höhle vor: Fissidens minutulus.

\section{SUMMARY}

The vegetation of the Forrás and István caves at Lillafüred in Hungary is composed of algae, micro- and macrofungi and mosses. The algae in both caves are represented by unicellular Cyanophyta and Chlorophyta with small species numbers. The macrofungi are Coprinus and Polyporacea spp. while it was impossible to identify the microfungi. The moss flora is richly developed and it can be supposed to represent a secondary vegetation at the artificially illuminated places of the caves. In Forrás cave 7, and in István cave 15 different mosses were found, only 3 of which proved to be common to both caves: Rhynchostegium murale, Eucladium verticillatum and Pohlia sp. A rare and interesting species: Fissidens minutulus occurred at several localities in Forrás cave.

\section{LITERATUR}

Boros, A. (1935) - Die Vegetation der Eishöhlen von Szilice und Barka. Bot. Közl. $32: 104-114$.

Claus, G. (1955) - Algae and their Mode of Life in the Baradla Cave at Aggtelek. Acta Bot. Acad. Sci. Hung. 2:1-27.

- (1959) - Concerning the Algal Flora of the Peace Cave at Aggtelek in Hungary. Proc. IX. Internat. Bot. Congr. 2A:75. 
Claus, G. (1962 a) - Beiträge zur Kenntnis der Algenflora der Abaligeter Höhle. Hydrobiologia 19:192-222.

- $(1962 \mathrm{~b})$ - Data on the Ecology of the Algae of Peace Cave in Hungary. Nova Hedwigia. 4:55-80.

FeHÉR, D. (1954) - Talajbiológia.:1-1264. Budapest.

GebHARDT, A. (1934) - Az Abaligeti-barlang élövilága:1-196. Budapest.

JAK UCS, L. (1952) - Az Aggteleki Cseppköbarlang.: 1-120. Budapest.

Kessler, H. (1953) - A lillafüredi Anna-barlang forrásai. Hidrol. Közl. $33: 16-22$.

Kessler, H., und Megay, G. (1961) - Lillafüred barlangjai.:1-82. Miskolc.

KoL, E. (1957) - Algological Investigations in the Ice-cave of the Sátormountain. Bot. Közl. 47:43-51.

Palık, P. (1960a) - Über die Algenwelt der Höhlen. Hidrol. Közl. 41: 417-422.

- (1960 b) - A New Blue-green Alga From the Cave Baradla Near Aggtelek. Ann. Univ. Sci. Budapestinensis. Sec. Biol. 3:275-285.

Suba, E. (1957) - Die Algen der Pálvölgyer Höhle in Ungarn. Verh. Zool.Bot. Ges. Wien. 97:97-109.

Szabó, G. (1963) - Angaben zum Mikroklima der Höhlen bei Lillafüred. Acta Climat. Szeged. 2-3(1-4):13-31.

\section{ERKLÄRUNG DER TAFEL 106 (1)}

Fig. 1: Panorama-Bild von Lillafüred.

Fig. 2: Eingang der István-Höhle.

Fig. 3: Moose in der István-Höhle.

Fig. 4: Massenhaftes Pflanzenvorkommen auf Stalaktiten in der IstvánHöhle.

Fig. 5: Massenhaftes Pflanzenvorkommen auf Stalagmiten in der IstvánHöhle.

Fig. 6: Algen- und Mikropilzbelag auf den Tropfsteinen in der Forrás-Höhle.

Fig. 7: Reiche Moosflora um die Lampe herum in der Forrás-Höhle. 

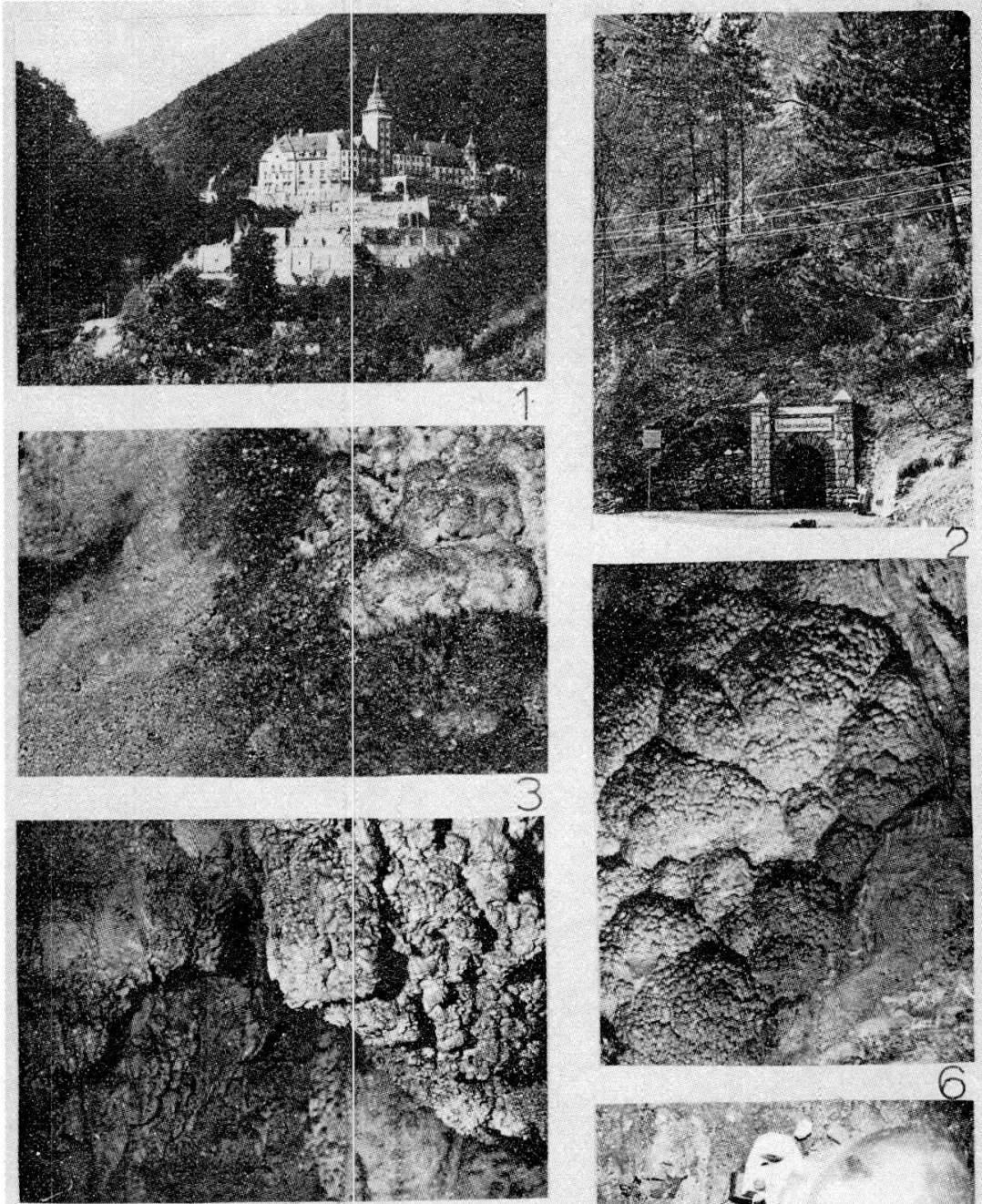

3
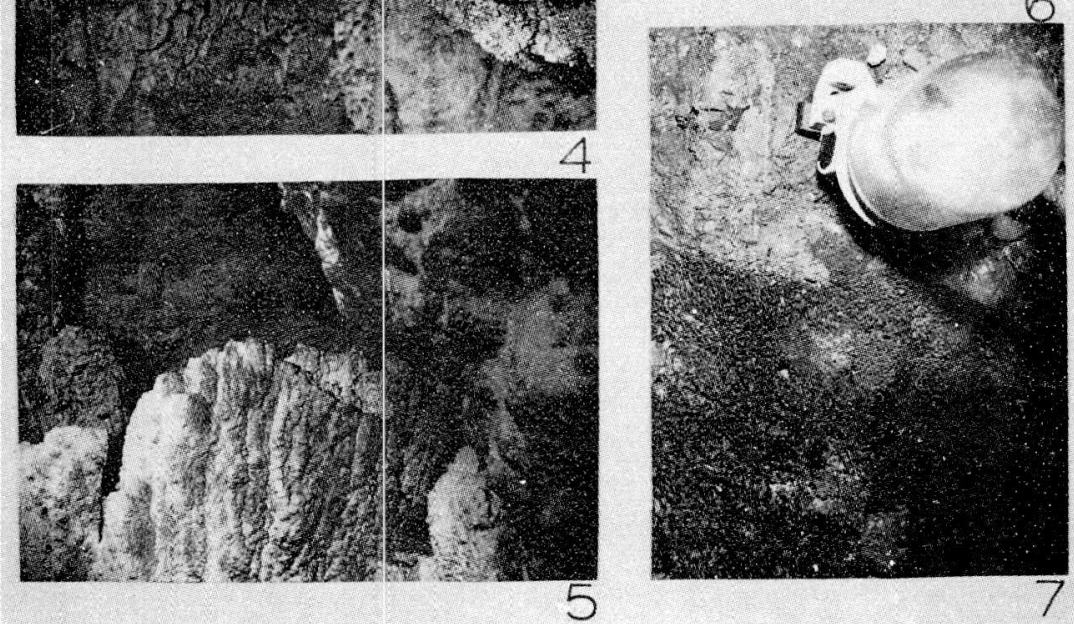